\title{
A fixed point method in dynamic processes for a class of elastic-viscoplastic materials
}

\author{
by A. Amassad (Perpignan)
}

\begin{abstract}
Two problems are considered describing dynamic processes for a class of rate-type elastic-viscoplastic materials with or without internal state variable. The existence and uniqueness of the solution is proved using classical results of linear elasticity theory together with a fixed point method.
\end{abstract}

1. Introduction. This work concerns two initial and boundary value problems describing dynamic processes for materials modeled by rate-type constitutive equations of the form

$$
\dot{\sigma}=\mathcal{E} \dot{\varepsilon}+G(\sigma, \varepsilon)
$$

or

$$
\dot{\sigma}=\mathcal{E} \dot{\varepsilon}+G(\sigma, \varepsilon, \kappa)
$$

where $\sigma$ is the stress tensor, $\varepsilon$ is the linearized strain tensor and $\kappa$ is an internal state variable. (In (1.1), (1.2) and everywhere in this paper the dot above a quantity represents the time derivative of that quantity). Such equations are used in order to model the behaviour of real bodies like rubbers, metals, rocks and so on (see for example Cristescu and Suliciu [1]). Existence and uniqueness results for quasistatic problems involving (1.1) and (1.2) were obtained by Ionescu and Sofonea [5] and by Djabi and Sofonea [2]. In [5], a Cauchy-Lipschitz method is used while in [2] fixed point arguments are utilized. Dynamic problems for models of the form (1.1) and (1.2) were already studied by Ionescu [4] and by Ionescu and Sofonea [5] using semigroup arguments.

The aim of this paper is to establish some results of [5] using only classical results of linear elasticity theory together with a fixed point technique. So,

1991 Mathematics Subject Classification: 73E60, 73E50, 73E25, 47H19.

Key words and phrases: viscoplasticity, dynamic processes, Galerkin method, fixed point, internal state variable. 
the fixed point method already used for quasistatic problem in [2] is here adopted in the case of a dynamic problem.

The paper is structured as follows: in Section 2, two mechanical problems $\left(P_{1}\right)$ and $\left(P_{2}\right)$ are stated and some preliminaries are given. In Section 3 we prove an existence and uniqueness result for the problem $\left(P_{1}\right)$ (Theorem 3.1) and finally in Section 4 we use the same technique in order to obtain an existence and uniqueness result for the problem $\left(P_{2}\right)$ (Theorem 4.1).

2. Statements of problems and preliminaries. Let $\Omega \subset \mathbb{R}^{N}$ be a bounded domain with a smooth boundary $\Gamma=\partial \Omega$ and let $\Gamma_{1}$ be an open subset of $\Gamma$. We denote by $\Gamma_{2}=\Gamma \backslash \bar{\Gamma}_{1}$ and let $T>0$ be a time interval.

Let us consider the following dynamic problem:

Problem $\left(P_{1}\right)$. Find the displacement function $u:[0, T] \times \Omega \rightarrow \mathbb{R}^{N}$ and the stress function $\sigma:[0, T] \times \Omega \rightarrow \mathcal{S}_{N}$ such that

$$
\begin{gathered}
\varrho \ddot{u}=\operatorname{div} \sigma+b \quad \text { in } \Omega \times(0, T), \\
\dot{\sigma}=\mathcal{E} \varepsilon(\dot{u})+G(\sigma, \varepsilon(u)) \quad \text { in } \Omega \times(0, T), \\
u=g \quad \text { on } \Gamma_{1} \times(0, T), \\
\sigma \nu=h \quad \text { on } \Gamma_{2} \times(0, T), \\
u(0)=u_{0}, \quad \dot{u}(0)=v_{0}, \quad \sigma(0)=\sigma_{0} \quad \text { in } \Omega .
\end{gathered}
$$

Here $\mathcal{S}_{N}$ is the set of second order symmetric tensors in $\mathbb{R}^{N}, \varepsilon(u)=\frac{1}{2}(\nabla u+$ $\nabla^{T} u$ ) defines the small strain tensor and $\nu$ is the exterior unit normal at $\Gamma$.

This problem represents a dynamic problem for rate-type models of the form (2.2) in which $\mathcal{E}$ is a fourth order tensor and $G$ is a given constitutive function. In the motion equation (2.1), $\varrho: \Omega \rightarrow \mathbb{R}_{+}$is the mass density, $\operatorname{div} \sigma$ represents the divergence of the vector-valued function $\sigma$ and $b:[0, T] \times \Omega \rightarrow$ $\mathbb{R}^{N}$ is the given body force. The functions $g$ and $h$ in (2.3), (2.4) are the given boundary data and finally the functions $u_{0}, v_{0}, \sigma_{0}$ in $(2.5)$ are the initial data.

Let $M$ be a natural number. We also consider the following dynamic problem defined as follows:

Problem $\left(P_{2}\right)$. Find the displacement function $u:[0, T] \times \Omega \rightarrow \mathbb{R}^{N}$, the stress function $\sigma:[0, T] \times \Omega \rightarrow \mathcal{S}_{N}$ and the internal state variable $\kappa:[0, T] \times \Omega \rightarrow \mathbb{R}^{M}$ such that

$$
\begin{gathered}
\varrho \ddot{u}=\operatorname{div} \sigma+b \quad \text { in } \Omega \times(0, T), \\
\dot{\sigma}=\mathcal{E} \varepsilon(\dot{u})+G(\sigma, \varepsilon(u), \kappa) \quad \text { in } \Omega \times(0, T), \\
\dot{\kappa}=\varphi(\sigma, \varepsilon(u), \kappa) \quad \text { in } \Omega \times(0, T), \\
u=g \quad \text { on } \Gamma_{1} \times(0, T), \\
\sigma \nu=h \quad \text { on } \Gamma_{2} \times(0, T),
\end{gathered}
$$




$$
u(0)=u_{0}, \quad \dot{u}(0)=v_{0}, \quad \sigma(0)=\sigma_{0}, \quad \kappa(0)=\kappa_{0} \quad \text { in } \Omega .
$$

This problem represents a dynamic problem for rate-type viscoplastic models of the form (2.7), (2.8) in which $\kappa$ is an internal state variable whose evolution is described by (2.8). Here $\mathcal{E}$ is a fourth order tensor while $G$ and $\varphi$ are given constitutive functions.

In (2.6)-(2.11) we used similar notation as in the problem (2.1)-(2.5): $\varrho$ is the mass density, $b$ is the given body force, $g$ and $h$ are the given boundary data and finally $u_{0}, v_{0}, \sigma_{0}$ and $\kappa_{0}$ are the initial data.

In the sequel, we denote by "." the inner product in the spaces $\mathbb{R}^{N}$, $\mathbb{R}^{M}$ and $\mathcal{S}_{N}$ and by $|\cdot|$ the Euclidean norm of these spaces. The following notation is also used:

$$
\begin{aligned}
H & =\left\{v=\left(v_{i}\right) \mid v_{i} \in L^{2}(\Omega), i=1, \ldots, N\right\}, \\
H_{1} & =\left\{v=\left(v_{i}\right) \mid v_{i} \in H^{1}(\Omega), i=1, \ldots, N\right\}, \\
\mathcal{H} & =\left\{\tau=\left(\tau_{i j}\right) \mid \tau_{i j}=\tau_{j i} \in L^{2}(\Omega), i, j=1, \ldots, N\right\}, \\
\mathcal{H}_{1} & =\{\tau \in \mathcal{H} \mid \operatorname{div} \tau \in H\}, \\
Y & =\left\{\kappa=\left(\kappa_{i}\right) \mid \kappa_{i} \in L^{2}(\Omega), i=1, \ldots, M\right\} .
\end{aligned}
$$

The spaces $H, H_{1}, \mathcal{H}, \mathcal{H}_{1}$ and $Y$ are real Hilbert spaces endowed with the canonical inner products denoted by $\langle\cdot, \cdot\rangle_{H},\langle\cdot, \cdot\rangle_{H_{1}},\langle\cdot, \cdot\rangle_{\mathcal{H}},\langle\cdot, \cdot\rangle_{\mathcal{H}_{1}}$ and $\langle\cdot, \cdot\rangle_{Y}$ given by

$$
\begin{gathered}
\langle u, v\rangle_{H}=\int_{\Omega} u_{i} v_{i} d x \quad \forall u, v \in H, \\
\langle\sigma, \tau\rangle_{\mathcal{H}}=\int_{\Omega} \sigma_{i j} \tau_{i j} d x \quad \forall \sigma, \tau \in \mathcal{H}, \\
\langle u, v\rangle_{H_{1}}=\langle u, v\rangle_{H}+\langle\varepsilon(u), \varepsilon(v)\rangle_{\mathcal{H}} \quad \forall u, v \in H_{1}, \\
\langle\sigma, \tau\rangle_{\mathcal{H}_{1}}=\langle\sigma, \tau\rangle_{\mathcal{H}}+\langle\operatorname{div} \sigma, \operatorname{div} \tau\rangle_{H} \quad \forall \sigma, \tau \in \mathcal{H}_{1}, \\
\langle\kappa, \theta\rangle_{Y}=\int_{\Omega} \kappa_{i} \theta_{i} d x \quad \forall \kappa, \theta \in Y .
\end{gathered}
$$

Let

$$
V=\left\{v \in H_{1} \mid \gamma_{1} v=0 \text { on } \Gamma_{1}\right\}
$$

be a closed subset of $H_{1}$ endowed with the norm of $H_{1}$ where $\gamma_{1}: H_{1} \rightarrow H_{\Gamma}$ is the trace map and $H_{\Gamma}=H^{1 / 2}(\Gamma)^{N}$.

Let us also recall that if $\tau \in \mathcal{H}_{1}$, then there exists $\gamma_{2} \tau \in H_{\Gamma}^{\prime}$ such that

$$
\left\langle\gamma_{2} \tau, \gamma_{1} v\right\rangle=\langle\tau, \varepsilon(v)\rangle_{\mathcal{H}}+\langle\operatorname{div} \tau, v\rangle_{H} \quad \forall v \in H_{1} .
$$

Here $H_{\Gamma}^{\prime}=\left[H^{-1 / 2}(\Gamma)\right]^{N}$ and $\langle\cdot, \cdot\rangle$ represents the duality map between $H_{\Gamma}^{\prime}$ and $H_{\Gamma}$. By $\left.\tau \nu\right|_{\Gamma_{2}}$ we mean the restriction of $\gamma_{2} \tau$ to $\gamma_{1}(V)$ and we denote 
by $\mathcal{V}$ the closed subspace of $\mathcal{H}_{1}$ defined by

$$
\mathcal{V}=\left\{\tau \in \mathcal{H}_{1} \mid \tau \nu=0 \text { on } \Gamma_{2}\right\} .
$$

So, using (2.12) we have

$$
\langle\tau, \varepsilon(v)\rangle_{\mathcal{H}}+\langle\operatorname{div} \tau, v\rangle_{H}=0 \quad \forall v \in V, \tau \in \mathcal{V} .
$$

Here we consider $V$ and $\mathcal{V}$ as real Hilbert spaces endowed with the inner products of $H_{1}$ and $\mathcal{H}_{1}$, respectively.

Finally, notice that if $X$ is one of the above real spaces, $k \in \mathbb{N}, 1 \leq p \leq \infty$ and $T>0$, we use the classical notation $L^{\infty}(0, T, X), W^{k, p}(0, T, X)$ and we denote by $|\cdot|_{\infty, X},|\cdot|_{k, p, X}$ the norms on the spaces $L^{\infty}(0, T, X)$ and $W^{k, p}(0, T, X)$, respectively.

3. The first existence and uniqueness result. In order to prove the existence and uniqueness of the solution for problem (2.1)-(2.5) the following assumptions will be used:

$\varrho \in L^{\infty}(\Omega)$ and there exists $\varrho_{0}>0$ such that $\varrho(x) \geq \varrho_{0}$ a.e. in $\Omega$,

$\mathcal{E}: \Omega \times \mathcal{S}_{N} \rightarrow \mathcal{S}_{N}$ is a symmetric and positive definite tensor, i.e.:

(a) $\mathcal{E}_{i j k h} \in L^{\infty}(\Omega) \forall i, j, k, h=1, \ldots, N$,

(b) $\mathcal{E} \sigma \cdot \tau=\sigma \cdot \mathcal{E} \tau \forall \sigma, \tau \in \mathcal{S}_{N}$, a.e. in $\Omega$,

(c) there exists $\alpha>0$ such that $\mathcal{E} \sigma \cdot \sigma \geq \alpha|\sigma|^{2} \forall \sigma \in \mathcal{S}_{N}$,

$G: \Omega \times \mathcal{S}_{N} \times \mathcal{S}_{N} \rightarrow \mathcal{S}_{N}$ and

(a) there exists $L>0$ such that $\left|G\left(x, \sigma_{1}, \varepsilon_{1}\right)-G\left(x, \sigma_{2}, \varepsilon_{2}\right)\right| \leq$ $L\left(\left|\sigma_{1}-\sigma_{2}\right|+\left|\varepsilon_{1}-\varepsilon_{2}\right|\right) \quad \forall \sigma_{1}, \sigma_{2}, \varepsilon_{1}, \varepsilon_{2} \in \mathcal{S}_{N}$, a.e. in $\Omega$,

(b) $x \mapsto G(x, \sigma, \varepsilon)$ is a measurable function with respect to the Lebesgue measure on $\Omega$, for all $\sigma, \varepsilon \in \mathcal{S}_{N}$,

(c) $x \mapsto G(x, 0,0) \in \mathcal{H}$,

$$
\begin{gathered}
b \in W^{1,1}(0, T, H), \\
g \in W^{3,1}\left(0, T, H_{\Gamma}\right), \\
h \in W^{2,1}\left(0, T, H_{\Gamma}^{\prime}\right), \\
u_{0} \in H_{1}, \quad v_{0} \in H_{1}, \quad \sigma_{0} \in \mathcal{H}_{1},
\end{gathered}
$$$$
u_{0}=g(0), \quad v_{0}=\dot{g}(0) \quad \text { on } \Gamma_{1}, \quad \sigma_{0} \nu=h(0) \quad \text { on } \Gamma_{2} \text {. }
$$

The main result of this section is the following:

Theorem 3.1. Let (3.1)-(3.8) hold. Then there is a unique solution $(u, \sigma)$ of (2.1)-(2.5) having the regularity $u \in W^{2, \infty}(0, T, H) \cap W^{1, \infty}\left(0, T, H_{1}\right)$, $\sigma \in W^{1, \infty}(0, T, \mathcal{H}) \cap L^{\infty}\left(0, T, \mathcal{H}_{1}\right)$.

The proof of Theorem 3.1 will be obtained in several steps. So, we suppose in the sequel that the assumptions of Theorem 3.1 are fulfilled. 

by

Let $\eta \in L^{\infty}(0, T, \mathcal{H})$ and let $z_{\eta} \in W^{1, \infty}(0, T, \mathcal{H})$ be the function given

$$
z_{\eta}(t)=\int_{0}^{t} \eta(s) d s+z_{0} \quad \text { for all } t \in[0, T]
$$

where

$$
z_{0}=\sigma_{0}-\mathcal{E} \varepsilon\left(u_{0}\right)
$$

We have:

LEMma 3.1. There exists a unique pair of functions $u_{\eta} \in W^{2, \infty}(0, T, H) \cap$ $W^{1, \infty}\left(0, T, H_{1}\right), \sigma_{\eta} \in W^{1, \infty}(0, T, \mathcal{H}) \cap L^{\infty}\left(0, T, \mathcal{H}_{1}\right)$ such that

$$
\begin{gathered}
\varrho \ddot{u}_{\eta}=\operatorname{div} \sigma_{\eta}+b \quad \text { in } \Omega \times(0, T), \\
\sigma_{\eta}=\mathcal{E} \varepsilon\left(u_{\eta}\right)+z_{\eta} \quad \text { in } \Omega \times(0, T), \\
u_{\eta}=g \quad \text { on } \Gamma_{1} \times(0, T), \\
\sigma_{\eta} \nu=h \quad \text { on } \Gamma_{2} \times(0, T), \\
u_{\eta}(0)=u_{0}, \quad \dot{u}_{\eta}(0)=v_{0}, \quad \sigma_{\eta}(0)=\sigma_{0} \quad \text { in } \Omega .
\end{gathered}
$$

Proof. The proof will be btained using standard arguments of elasticity as in [2]. However, for convenience of the reader we present here a sketch of the proof in which the Galerkin method is used.

Let $X$ be the product Hilbert space $X=H \times \mathcal{H}$ with inner product

$$
\langle x, y\rangle_{X}=\langle\varrho u, v\rangle_{H}+\left\langle\mathcal{E}^{-1} \sigma, \tau\right\rangle_{\mathcal{H}}
$$

for all $x=(u, \sigma), y=(v, \tau) \in X$. Let $D(A)=V \times \mathcal{V} \subset X$. We also use the notation $[D(A)]$ for the real Hilbert space $D(A)$ endowed with the canonical inner product of the space $H_{1} \times \mathcal{H}_{1}$ and let $A: X \supset D(A) \rightarrow X$ be given by

$$
A x=\left(\varrho^{-1} \operatorname{div} \sigma, \mathcal{E} \varepsilon(u)\right) \forall x=(u, \sigma) \in X .
$$

By (3.5) and (3.6), there exist $\widetilde{u} \in W^{3,1}\left(0, T, H_{1}\right)$ and $\widetilde{\sigma} \in W^{2,1}\left(0, T, \mathcal{H}_{1}\right)$ such that

$$
\widetilde{u}=g \quad \text { on } \Gamma_{1} \times(0, T), \quad \widetilde{\sigma} \nu=h \quad \text { on } \Gamma_{2} \times(0, T) .
$$

Let us introduce the following notation:

$$
\begin{gathered}
\bar{u}_{\eta}=u_{\eta}-\widetilde{u}, \quad \bar{v}_{\eta}=\dot{\bar{u}}_{\eta}, \quad \bar{\sigma}_{\eta}=\sigma_{\eta}-\widetilde{\sigma}, \\
\bar{u}_{0}=u_{0}-\widetilde{u}(0), \quad \bar{v}_{0}=v_{0}-\dot{\tilde{u}}(0), \quad \bar{\sigma}_{0}=\sigma_{0}-\widetilde{\sigma}(0), \\
\quad x_{\eta}=\left(\bar{v}_{\eta}, \bar{\sigma}_{\eta}\right), \\
\quad x_{0}=\left(\bar{v}_{0}, \bar{\sigma}_{0}\right), \\
\left\{\begin{array}{l}
a_{\eta}:[0, T] \rightarrow X, \\
a_{\eta}(t)=\left(\varrho^{-1} b(t)-\ddot{\widetilde{u}}(t)+\varrho^{-1} \operatorname{div} \widetilde{\sigma}(t), \eta+\mathcal{E} \varepsilon(\dot{\tilde{u}}(t))-\dot{\tilde{\sigma}}(t)\right) .
\end{array}\right.
\end{gathered}
$$


It is easy to verify that the pair $\left(u_{\eta}, \sigma_{\eta}\right)$ is a solution of $(3.11)-(3.15)$ having the regularity $u_{\eta} \in W^{2, \infty}(0, T, H) \cap W^{1, \infty}\left(0, T, H_{1}\right), \sigma_{\eta} \in W^{1, \infty}(0, T, \mathcal{H})$ $\cap L^{\infty}\left(0, T, \mathcal{H}_{1}\right)$ if and only if

$$
\begin{gathered}
x_{\eta} \in W^{1, \infty}(0, T, X) \cap L^{\infty}(0, T,[D(A)]), \\
\dot{x}_{\eta}(t)=A x_{\eta}(t)+a_{\eta}(t), \\
x_{\eta}(0)=x_{0} .
\end{gathered}
$$

Let $\left(e_{m}\right)_{m}$ be a base of $X$ and for a given $m \in \mathbb{N}$ let $X_{m}$ denote the linear subspace of $X$ spanned by the vectors $\left\{e_{1}, \ldots, e_{m}\right\}$. Also let $\left(x_{0}^{m}\right)_{m \in \mathbb{N}} \subset X$ be such that

$$
x_{0}^{m} \in X_{m} \quad \forall m \in \mathbb{N}, \quad x_{0}^{m} \rightarrow x_{0} \quad \text { as } m \rightarrow \infty \quad \text { in } X .
$$

By standard arguments of ordinary differential equations there exists $x_{\eta}^{m} \in C^{1}\left(0, T, X_{m}\right)$ such that

$$
\begin{gathered}
\left\langle\dot{x}_{\eta}^{m}(t), y\right\rangle_{X}=\left\langle A x_{\eta}^{m}(t), y\right\rangle_{X}+\left\langle a_{\eta}(t), y\right\rangle_{X} \forall y \in X_{m}, t \in[0, T], \\
x_{\eta}^{m}(0)=x_{0}^{m} .
\end{gathered}
$$

From (3.25), by differentiating with respect to $t$ we have

$$
\left\{\begin{array}{l}
\left\langle\ddot{x}_{\eta}^{m}(t), y\right\rangle_{X}=\left\langle A \dot{x}_{\eta}^{m}(t), y\right\rangle_{X}+\left\langle\dot{a}_{\eta}(t), y\right\rangle_{X} \\
\forall y \in X_{m}, \text { a.e. } t \in(0, T) .
\end{array}\right.
$$

From (3.25)-(3.27) it follows that

$$
\begin{aligned}
& \left(x_{\eta}^{m}\right)_{m} \text { is a bounded sequence in } L^{\infty}(0, T, X), \\
& \left(\dot{x}_{\eta}^{m}\right)_{m} \text { is a bounded sequence in } L^{\infty}(0, T, X) .
\end{aligned}
$$

From (3.28)-(3.29) and using standard compactness arguments we see that there exists an element $x_{\eta} \in W^{1, \infty}(0, T, X)$ such that, passing to a subsequence again denoted by $\left(x_{\eta}^{m}\right)_{m}$, we have

$$
\begin{aligned}
& x_{\eta}^{m} \rightarrow x_{\eta} \quad \text { in } L^{\infty}(0, T, X) \text { weak* } \\
& \dot{x}_{\eta}^{m} \rightarrow \dot{x}_{\eta} \quad \text { in } L^{\infty}(0, T, X) \text { weak* }
\end{aligned}
$$

From (3.24)-(3.26), (3.30) and (3.31) it results that $x_{\eta}$ is a solution of (3.22)(3.23) having the regularity (3.21).

Using (3.3), we can define the operator $\Lambda: L^{\infty}(0, T, \mathcal{H}) \rightarrow L^{\infty}(0, T, \mathcal{H})$ as follows:

$$
\Lambda \eta(t)=G\left(\sigma_{\eta}(t), \varepsilon\left(u_{\eta}(t)\right)\right)
$$

for all $t \in[0, T]$ and $\eta \in L^{\infty}(0, T, \mathcal{H})$ where $\left(u_{\eta}, \sigma_{\eta}\right)$ denotes the solution obtained in Lemma 3.1.

We have the following result:

Lemma 3.2. The operator $\Lambda$ has a unique fixed point $\eta^{*} \in L^{\infty}(0, T, \mathcal{H})$. 
Proof. Let $\eta_{1}, \eta_{2} \in L^{\infty}(0, T, \mathcal{H})$. Using (3.9)-(3.15) we may define the functions $z_{\eta_{i}}, u_{\eta_{i}}$ and $\sigma_{\eta_{i}}$ for $i=1,2$. For simplicity we set: $z_{\eta_{1}}=z_{1}, z_{\eta_{2}}=$ $z_{2}, u_{\eta_{1}}=u_{1}, u_{\eta_{2}}=u_{2}, \sigma_{\eta_{1}}=\sigma_{1}, \sigma_{\eta_{2}}=\sigma_{2}$ and let $v_{1}=\dot{u}_{1}, v_{2}=\dot{u}_{2}$.

We also introduce the following notation: $z=z_{1}-z_{2}, u=u_{1}-u_{2}$, $v=\dot{u}, \sigma=\sigma_{1}-\sigma_{2}, \eta=\eta_{1}-\eta_{2}$.

From (3.11)-(3.15) we see that $u, v, \sigma$ satisfy:

$$
\begin{gathered}
\dot{u}=v \quad \text { in } \Omega \times(0, T), \\
\varrho \dot{v}=\operatorname{div} \sigma \quad \text { in } \Omega \times(0, T), \\
\dot{\sigma}=\mathcal{E} \varepsilon(v)+\eta \quad \text { in } \Omega \times(0, T), \\
u=0 \quad \text { on } \Gamma_{1} \times(0, T), \\
\sigma \nu=0 \quad \text { on } \Gamma_{2} \times(0, T), \\
u(0)=0, \quad v(0)=0, \quad \sigma(0)=0 \quad \text { in } \Omega .
\end{gathered}
$$

It results that $u(t) \in V$ and $\sigma(t) \in \mathcal{V}$ for all $t \in[0, T]$. So, from (2.13), (3.2) and (3.34)-(3.37) it follows that

$$
\left\langle\sigma, \mathcal{E}^{-1} \dot{\sigma}\right\rangle_{\mathcal{H}}+\langle\varrho \dot{v}, v\rangle_{H}=\left\langle\sigma, \mathcal{E}^{-1} \eta\right\rangle_{\mathcal{H}} \quad \text { a.e. on }(0, T) .
$$

Using now (3.38), after integration and some algebra we deduce

$$
\frac{1}{2}|\sigma(t)|_{\mathcal{H}}^{2}+\frac{1}{2}|v(t)|_{H}^{2} \leq C \int_{0}^{t}|\sigma(s)|_{\mathcal{H}}|\eta(s)|_{\mathcal{H}} d s \quad \forall t \in[0, T]
$$

hence we obtain

$$
\frac{1}{2}|\sigma(t)|_{\mathcal{H}}^{2} \leq C \int_{0}^{t}|\sigma(s)|_{\mathcal{H}}|\eta(s)|_{\mathcal{H}} d s \quad \forall t \in[0, T] .
$$

Here and everywhere in this paper $C>0$ represents strictly positive generic constants which may depend only on $\Omega, \Gamma_{1}, \Gamma_{2}, \mathcal{E}$ and $G$ and do not depend on time or the input data $b, g, h, u_{0}$ and $\sigma_{0}$.

Using (3.39) and a Gronwall type lemma we obtain

$$
|\sigma(t)|_{\mathcal{H}} \leq C \int_{0}^{t}|\eta(s)|_{\mathcal{H}} d s \quad \forall t \in[0, T],
$$

and, from (3.32) and (3.3) it follows that

(3.41) $\left|\Lambda \eta_{1}(t)-\Lambda \eta_{2}(t)\right|_{\mathcal{H}} \leq L\left(\left|\sigma_{1}(t)-\sigma_{2}(t)\right|_{\mathcal{H}}+\left|\varepsilon\left(u_{1}(t)\right)-\varepsilon\left(u_{2}(t)\right)\right|_{\mathcal{H}}\right)$

for all $t \in[0, T]$. Since

$$
\varepsilon\left(u_{i}\right)=\mathcal{E}^{-1} \sigma_{i}-\mathcal{E}^{-1} z_{i} \quad \text { for } i=1,2 \text { and } t \in[0, T],
$$

from (3.41) and (3.2) we deduce that

$(3.42)\left|\Lambda \eta_{1}(t)-\Lambda \eta_{2}(t)\right|_{\mathcal{H}} \leq C\left(\left|\sigma_{1}(t)-\sigma_{2}(t)\right|_{\mathcal{H}}+\left|z_{1}(t)-z_{2}(t)\right|_{\mathcal{H}}\right) \forall t \in[0, T]$. 
Then, from (3.9), (3.40) and (3.42) it results that

$$
\left|\Lambda \eta_{1}(t)-\Lambda \eta_{2}(t)\right|_{\mathcal{H}} \leq C \int_{0}^{t}\left|\eta_{1}(s)-\eta_{2}(s)\right|_{\mathcal{H}} d s \quad \forall t \in[0, T] .
$$

By recurrence we can verify that this inequality leads to

$$
\left|\Lambda^{p} \eta_{1}-\Lambda^{p} \eta_{2}\right|_{\infty, \mathcal{H}} \leq \frac{C^{p} T^{p}}{p !}\left|\eta_{1}-\eta_{2}\right|_{\infty, \mathcal{H}} \quad \forall p \in \mathbb{N},
$$

which implies that, for $p$ large enough, a power $\Lambda^{p}$ of $\Lambda$ is a contraction in $L^{\infty}(0, T, \mathcal{H})$. So, there exists a unique $\eta^{*} \in L^{\infty}(0, T, \mathcal{H})$ such that $\Lambda^{p} \eta^{*}=$ $\eta^{*}$. Moreover, $\eta^{*}$ is the unique fixed point of $\Lambda$.

Proof of Theorem 3.1. The existence part. Let $\eta^{*} \in L^{\infty}(0, T, \mathcal{H})$ be the fixed point of $\Lambda$ and let $u_{\eta^{*}} \in W^{2, \infty}(0, T, H) \cap W^{1, \infty}\left(0, T, H_{1}\right), \sigma_{\eta^{*}} \in$ $W^{1, \infty}(0, T, \mathcal{H}) \cap L^{\infty}\left(0, T, \mathcal{H}_{1}\right)$ be the functions given by Lemma 3.1 for $\eta=\eta^{*}$. We shall prove that $\left(u_{\eta^{*}}, \sigma_{\eta^{*}}\right)$ is a solution for the problem $\left(P_{1}\right)$. For this, we have to prove (2.2). This equality follows from (3.12), (3.9) and (3.32) since

$$
\left\{\begin{array}{l}
\dot{\sigma}_{\eta^{*}}(t)=\mathcal{E} \varepsilon\left(\dot{u}_{\eta^{*}}(t)\right)+\dot{z}_{\eta^{*}}(t), \\
\dot{z}_{\eta^{*}}(t)=\eta^{*}(t)=\Lambda \eta^{*}(t)=G\left(\sigma_{\eta^{*}}(t), \varepsilon\left(u_{\eta^{*}}(t)\right)\right),
\end{array}\right.
$$

The uniqueness part. In order to prove the uniqueness part in Theorem 3.1, let $\left(u_{\eta^{*}}, \sigma_{\eta^{*}}\right)$ be the solution of $\left(P_{1}\right)$ obtained above and let $(u, \sigma)$ be another solution of $\left(P_{1}\right)$ having the same regularity, i.e. $u \in$ $W^{2, \infty}(0, T, H) \cap W^{1, \infty}\left(0, T, H_{1}\right), \sigma \in W^{1, \infty}(0, T, \mathcal{H}) \cap L^{\infty}\left(0, T, \mathcal{H}_{1}\right)$. We denote by $\eta \in L^{\infty}(0, T, \mathcal{H})$ the function defined by

$$
\eta(t)=G(\sigma(t), \varepsilon(u(t))) \quad \forall t \in[0, T]
$$

and let $z_{\eta} \in W^{1, \infty}(0, T, \mathcal{H})$ be given by (3.9), (3.10). Since from (2.1)(2.5) it results that $(u, \sigma)$ satisfy $(3.11)-(3.15)$ and since this problem has a unique solution $u_{\eta} \in W^{2, \infty}(0, T, H) \cap W^{1, \infty}\left(0, T, H_{1}\right), \sigma_{\eta} \in W^{1, \infty}(0, T, \mathcal{H}) \cap$ $L^{\infty}\left(0, T, \mathcal{H}_{1}\right)$, it results that

$$
u=u_{\eta}, \quad \sigma=\sigma_{\eta} .
$$

Using now (3.32), (3.43) and (3.44) we get $\Lambda \eta=\eta$ and by the uniqueness of the fixed point of $\Lambda$ we have

$$
\eta=\eta^{*} \text {. }
$$

The uniqueness part of Theorem 3.1 is now a consequence of (3.44) and (3.45).

4. The second existence and uniqueness result. In order to study the problem (2.6)-(2.11) we also consider the following assumptions: 
(4.1) $\quad G: \Omega \times \mathcal{S}_{N} \times \mathcal{S}_{N} \times \mathbb{R}^{M} \rightarrow \mathcal{S}_{N}$ and

(a) there exists $L>0$ such that $\left|G\left(x, \sigma_{1}, \varepsilon_{1}, \kappa_{1}\right)-G\left(x, \sigma_{2}, \varepsilon_{2}, \kappa_{2}\right)\right|$ $\leq L\left(\left|\sigma_{1}-\sigma_{2}\right|+\left|\varepsilon_{1}-\varepsilon_{2}\right|+\left|\kappa_{1}-\kappa_{2}\right|\right) \quad \forall \sigma_{1}, \sigma_{2}, \varepsilon_{1}, \varepsilon_{2} \in \mathcal{S}_{N}$, $\kappa_{1}, \kappa_{2} \in \mathbb{R}^{M}$ a.e. in $\Omega$,

(b) $x \mapsto G(x, \sigma, \varepsilon, \kappa)$ is a measurable function with respect to the Lebesgue measure on $\Omega$, for all $\sigma, \varepsilon \in \mathcal{S}_{N}, \kappa \in \mathbb{R}^{M}$,

(c) $x \mapsto G(x, 0,0,0) \in \mathcal{H}$,

$\varphi: \Omega \times \mathcal{S}_{N} \times \mathcal{S}_{N} \times \mathbb{R}^{M} \rightarrow \mathbb{R}^{M}$ and

(a) there exists $L^{\prime}>0$ such that $\left|\varphi\left(x, \sigma_{1}, \varepsilon_{1}, \kappa_{1}\right)-\varphi\left(x, \sigma_{2}, \varepsilon_{2}, \kappa_{2}\right)\right|$ $\leq L^{\prime}\left(\left|\sigma_{1}-\sigma_{2}\right|+\left|\varepsilon_{1}-\varepsilon_{2}\right|+\left|\kappa_{1}-\kappa_{2}\right|\right) \forall \sigma_{1}, \sigma_{2}, \varepsilon_{1}, \varepsilon_{2} \in \mathcal{S}_{N}$, $\kappa_{1}, \kappa_{2} \in \mathbb{R}^{M}$ a.e. in $\Omega$,

(b) $x \mapsto \varphi(x, \sigma, \varepsilon, \kappa)$ is a measurable function with respect to the Lebesgue measure on $\Omega$, for all $\sigma, \varepsilon \in \mathcal{S}_{N}, \kappa \in \mathbb{R}^{M}$,

(c) $x \mapsto \varphi(x, 0,0,0) \in Y$,

(4.3) $\kappa_{0} \in Y$.

The main result of this section is the following:

Theorem 4.1. Let (3.1), (3.2), (3.4)-(3.8), (4.1)-(4.3) hold. Then there exists a unique solution of the problem (2.6)-(2.11) having the regularity $u \in W^{2, \infty}(0, T, H) \cap W^{1, \infty}\left(0, T, H_{1}\right), \sigma \in W^{1, \infty}(0, T, \mathcal{H}) \cap L^{\infty}\left(0, T, \mathcal{H}_{1}\right)$ and $\kappa \in W^{1, \infty}(0, T, Y)$.

Proof. We use a similar technique to the proof of Theorem 3.1. Let $Z$ be the product Hilbert space $Z=\mathcal{H} \times Y$, let $\eta=\left(\eta^{1}, \eta^{2}\right) \in L^{\infty}(0, T, Z)$, let $z_{\eta}=\left(z_{\eta}{ }^{1}, z_{\eta}{ }^{2}\right) \in W^{1, \infty}(0, T, Z)$ be the function given by

$$
z_{\eta}(t)=\int_{0}^{t} \eta(s) d s+z_{0} \quad \text { for all } t \in[0, T]
$$

where

$$
z_{0}=\left(\sigma_{0}-\mathcal{E} \varepsilon\left(u_{0}\right), \kappa_{0}\right)
$$

Using arguments similar to Lemma 3.1 we obtain the existence and uniqueness of $u_{\eta} \in W^{2, \infty}(0, T, H) \cap W^{1, \infty}\left(0, T, H_{1}\right)$ and $\sigma_{\eta} \in W^{1, \infty}(0, T, \mathcal{H}) \cap$ $L^{\infty}\left(0, T, \mathcal{H}_{1}\right)$ such that

$$
\begin{gathered}
\varrho \ddot{u}_{\eta}=\operatorname{div} \sigma_{\eta}+b \quad \text { in } \Omega \times(0, T), \\
\sigma_{\eta}=\mathcal{E} \varepsilon\left(u_{\eta}\right)+z_{\eta}^{1} \quad \text { in } \Omega \times(0, T), \\
u_{\eta}=g \quad \text { on } \Gamma_{1} \times(0, T), \\
\sigma_{\eta} \nu=h \quad \text { on } \Gamma_{2} \times(0, T), \\
u_{\eta}(0)=u_{0}, \quad \dot{u}_{\eta}(0)=v_{0}, \quad \sigma_{\eta}(0)=\sigma_{0} \quad \text { in } \Omega .
\end{gathered}
$$


Let $\kappa_{\eta} \in W^{1, \infty}(0, T, Y)$ be the function defined by

$$
\kappa_{\eta}=z_{\eta}^{2}
$$

Using (4.1) and (4.2) we can define the operator $\Lambda: L^{\infty}(0, T, Z) \rightarrow$ $L^{\infty}(0, T, Z)$ as follows:

$$
\Lambda \eta(t)=\left(G\left(\sigma_{\eta}(t), \varepsilon\left(u_{\eta}(t)\right), \kappa_{\eta}(t)\right), \varphi\left(\sigma_{\eta}(t), \varepsilon\left(u_{\eta}(t)\right), \kappa_{\eta}(t)\right)\right)
$$

for all $t \in[0, T]$ and $\eta \in L^{\infty}(0, T, Z)$.

We shall prove that the operator $\Lambda$ has a unique fixed point $\eta^{*} \in$ $L^{\infty}(0, T, Z)$.

Indeed, let $\eta_{1}=\left(\eta_{1}^{1}, \eta_{1}^{2}\right), \eta_{2}=\left(\eta_{2}^{1}, \eta_{2}^{2}\right) \in L^{\infty}(0, T, Z)$. Using (4.4), (4.6)(4.11) we may define the functions $z_{\eta_{i}}, u_{\eta_{i}}, \sigma_{\eta_{i}}$ and $\kappa_{\eta_{i}}$ for $i=1,2$. For simplicity we define $z_{\eta_{1}}=z_{1}, z_{\eta_{2}}=z_{2}, u_{\eta_{1}}=u_{1}, u_{\eta_{2}}=u_{2}, \sigma_{\eta_{1}}=\sigma_{1}, \sigma_{\eta_{2}}=$ $\sigma_{2}, \kappa_{\eta_{1}}=\kappa_{1}, \kappa_{\eta_{2}}=\kappa_{2}$. We also introduce the following notation: $\eta^{1}=$ $\eta_{1}^{1}-\eta_{2}^{1}, \eta^{2}=\eta_{1}^{2}-\eta_{2}^{2}, z^{1}=z_{1}^{1}-z_{2}^{1}, u=u_{1}-u_{2}, v=\dot{u}, \sigma=\sigma_{1}-\sigma_{2}$, $\kappa=\kappa_{1}-\kappa_{2}$.

From (4.6)-(4.9) we get

$$
\begin{gathered}
\varrho(\ddot{u})=\operatorname{div} \sigma \quad \text { in } \Omega \times(0, T), \\
\sigma=\mathcal{E} \varepsilon(u)+z^{1} \quad \text { in } \Omega \times(0, T), \\
u=0 \quad \text { on } \Gamma_{1} \times(0, T), \\
\sigma \nu=0 \quad \text { on } \Gamma_{2} \times(0, T), \\
u(0)=0, \quad v(0)=0, \quad \sigma(0)=0 \quad \text { in } \Omega .
\end{gathered}
$$

From (2.13), (4.14)-(4.16) it follows that

$$
|\sigma(t)|_{\mathcal{H}} \leq C \int_{0}^{t}\left|\eta^{1}(s)\right|_{\mathcal{H}} d s \quad \forall t \in[0, T] .
$$

Since

$$
\varepsilon(u)=\mathcal{E}^{-1} \sigma-\mathcal{E}^{-1} z^{1} \quad \forall t \in[0, T]
$$

from (4.18) and (4.4) we have

$$
|\varepsilon(u(t))|_{\mathcal{H}} \leq C \int_{0}^{t}\left|\eta^{1}(s)\right|_{\mathcal{H}} d s \quad \forall t \in[0, T]
$$

and using (4.11) we obtain

$$
|\kappa(t)|_{Y} \leq C \int_{0}^{t}\left|\eta^{2}(s)\right|_{Y} d s \quad \forall t \in[0, T] .
$$

Using (4.12), (4.1) and (4.2) it follows that

$$
\left|\Lambda \eta_{1}(t)-\Lambda \eta_{2}(t)\right|_{Z} \leq C\left(|\sigma(t)|_{\mathcal{H}}+|\varepsilon(u(t))|_{\mathcal{H}}+|\kappa(t)|_{Y}\right) .
$$


Then from (4.21), (4.18)-(4.20) it results that

$$
\begin{aligned}
& \left|\Lambda \eta_{1}(t)-\Lambda \eta_{2}(t)\right|_{Z} \\
& \quad \leq C \int_{0}^{t}\left(\left|\eta_{1}^{1}(s)-\eta_{2}^{1}(s)\right|_{\mathcal{H}}+\left|\eta_{1}^{2}(s)-\eta_{2}^{2}(s)\right|_{Y}\right) d s \quad \text { for all } t \in[0, T]
\end{aligned}
$$

where $C$ depends only on $\Omega, \Gamma_{1}, \mathcal{E}, G$ and $\varphi$.

By recurrence we can verify that this inequality leads to

$$
\left|\Lambda^{p} \eta_{1}-\Lambda^{p} \eta_{2}\right|_{\infty, Z} \leq \frac{C^{p} T^{p}}{p !}\left|\eta_{1}-\eta_{2}\right|_{\infty, Z} \quad \forall p \in \mathbb{N},
$$

which implies that, for $p$ large enough, a power $\Lambda^{p}$ of $\Lambda$ is a contraction in $L^{\infty}(0, T, Z)$. Then, there exists a unique $\eta^{*} \in L^{\infty}(0, T, Z)$ such that $\Lambda^{p} \eta^{*}=\eta^{*}$. Moreover, $\eta^{*}$ is the unique fixed point of $\Lambda$.

Using now (4.4)-(4.11) and (4.12) we see that $u_{\eta^{*}} \in W^{2, \infty}(0, T, H) \cap$ $W^{1, \infty}\left(0, T, H_{1}\right), \sigma_{\eta^{*}} \in W^{1, \infty}(0, T, \mathcal{H}) \cap L^{\infty}\left(0, T, \mathcal{H}_{1}\right), \kappa_{\eta^{*}} \in W^{1, \infty}(0, T, Y)$ is a solution of $(2.6)-(2.11)$.

The uniqueness part of Theorem 4.1 follows from the uniqueness of the fixed point of $\Lambda$ using the same technique as in the proof of Theorem 3.1 or by standard arguments for evolution equations.

\section{References}

[1] N. Cristescu and I. Suliciu, Viscoplasticity, Martius Nijhoff and Editura Tehnica, Bucarest, 1982.

[2] S. Djabi and M. Sofonea, A fixed point method in quasistatic rate-type viscoplasticty, Appl. Math. Comput. Sci., 1993.

[3] G. Duvaut et J. L. Lions, Les Inéquations en Mécanique et en Physique, Dunod, Paris, 1972.

[4] I. R. Ionescu, Dynamic processes for a class of elastic-viscoplastic materials, Stud. CBRC Mat., Bucureşti, 1992.

[5] I. R. Ionescu and M. Sofonea, Functional and Numerical Methods in Viscoplasticity, Oxford Univ. Press, Oxford, 1993.

Department of Mathematics

University of Perpignan

52, Avenue de Villeneuve

66860 Perpignan Cedex, France

E-mail: amassad@univ-perp.fr 